Multi-Media Session 2793

\title{
Selecting Communications Technology for Delivering Continuing Professional Education (CPE) Programs
}

\author{
Peter J. Graybash, Jr. \\ Pennsylvania State University
}

\begin{abstract}
With the convergence of major shifts in economy and society, competency development programs for professional employees in American corporations are increasing in demand and availability. Communications technologies now allow unprecedented linkages between Continuing Professional Engineering Educators (CPEE) and their organizational consumers. As development programs become more available, a multitude of decisions must be made concerning not just the equipment and technology issues, but also "human elements" relating to the teaching and learning and participation by organizational personnel. Not all decisions are made on economics.
\end{abstract}

The principal objective of this project is to identify the human elements addressed by Continuing Engineering Educators in high-tech industries as they decide on delivery systems for employee education. Information will be collected via a three-phase Delphi study of key decision-makers in a purposive sample of 23 leading high-tech Fortune 500 companies. The first phase of the study involves open-ended questions directed at a focus group. From the focus group comes a written survey questionnaire seeking to define the best match for delivery of competencies, the concerns, the reservations and the challenges. The final phase is telephone interviews for in-depth exploration. It is expected that many of the themes that emerged from the study do not align with what adult educators emphasize in instructional design and delivery, suggesting a more holistic perspective on issues of program planning.

Significance to the field of CPEE is in the importance of human factors in decision-making, which are often denied or ignored. For the corporate educator concerned with instructional design and the learning environment, the impact of the delivery medium with attributed effort, ability, resistance, need to do well, and other items of social need and learner attitude will be better understood. If done well, decision quality will be improved

\section{Introduction}

For many, the "Information Age" arrived before we realized it, with the 1990s witnessing unparalleled growth and development of information technology. This has changed the world around us -- not only how we view it, but also how we interact and cope with it. Staying current in this rapidly evolving world is a considerable challenge for those seeking to enhance their value as employees, and expand their horizons as individuals. Communications technology has 
had a dual effect on us - driving the need for continuing education, and expanding our opportunities for obtaining it. Distance Education, for example, has been re-defined to describe the teaching-learning relationship in which participants interact through advanced communication technology.

Career competition with growing numbers of increasingly sophisticated fellow "experts" is on the minds and tongues of those in virtually every profession. Just as our appetite for continued career education grows, the diversity and sophistication of ways to deliver that education continues to expand. Therefore, it becomes complex, expensive, and difficult to pick from. We are not sure which of these delivery systems is the most effective. There are ways to measure effectiveness from an engineering standpoint, but a large portion of the choice is behaviorally oriented.

\section{Profession Awakens to the Need}

Continuing Professional Education is especially critical in the engineering profession, where knowledge has a generally accepted five-year half-life. That is, half of what a new graduate has learned is obsolete within five years. Engineers become obsolete, how do we get that overcome? The need for career-long continuing engineering education was formally recognized by individual engineers, industry experts, academicians and professional societies when a panel of the National Research Council recommended that the National Science Foundation (NSF) establish learning objectives for career-long engineering education.

In 1992,the NSF funded several engineering educational institutions to define the learning objectives, and to test and verify whether people learn more effectively in self-paced or collaborative learning environments. The result was the National Engineering Education Delivery System (NEEDS). Through NEEDS, the NSF demonstrated that it recognized the value of rapid response to specific business-related problems. Equally important, there was also a clear recognition that adults learn in different ways, with different attitudes and directions, celebrating self-identity and the development of full potentialities. Malcolm Knowles earlier had refined the art and science of teaching adults, coined the term "andragogy", and defined it as a lifelong process of continuing inquiry.

The development of Continuing Professional Education is a point in the evolution of the concept of andragogy that Malcolm Knowles described. The primary purpose is the same. CPE continues to help adult individuals satisfy their life needs and achieve their life goals (Knowles, 1980). Given the need for adult education, the question arises as just what is the most effective way to teach them. In recent years, research has shown many attempts to use the latest communications technology. There is continued high use of teleconferencing formats and multiple conferencing technologies, along with high enthusiasm for its use. However, preliminary research indicates that new systems have generally failed to be studied from the context of communications technology, while other research confirms industry managers' dissatisfaction with the current status of continuing education programs. From an instructional design perspective, what is the most effective mode of delivery? This raises the interesting question. In the next section, we'll consider one of the most popular areas in which technology has been growing fastest. Distance Education is being used by companies that do not want to release employees for long periods of 
time, don't want them away from the job, and don't want to spend money on unnecessary travel.

\section{Learning From A Distance}

Distance Education is a field in which the use of technology has been most prevalent and rapidly growing. Interest in telecommunicated distance education has been growing so rapidly that it is impossible to accurately document the many projects presently underway or being considered. Distance learning projects using communication technologies such as cable television, fiber optics, satellites, and microcomputer networking have opened multiple opportunities to coordinate schedules and share resources, thereby expanding curricular offerings and educational opportunities. A major factor in the growing interest in distance education is the ability to conduct live, real-time interaction between teachers and students.

Technology is often equated with "goodness", and almost always is assumed to be an improvement. But we don't know a lot about how Continuing Professional Educators go about making their technology selection decisions, or how they integrate individual human elements with organizational factors in the decision-making process.

When business organizations consider certain communication technologies, they focus largely on technical issues. They rarely or only marginally consider the non-technical or human elements ultimately influencing levels of acceptance and effectiveness of the chosen technologies on the teachers or their participants. Is the media selected on the basis of defined learning needs? Is the delivery system selected because it is more glamorous or more expensive? What are the criteria for selection? Furthermore, which human elements affect technology choices, and Why?

Consider, for example, the gap between educational needs and the education actually provided. The gap is wide, and even though much of it is a result of the technological age, communication technology and distance education may very well be the best means of bridging that gap.

\section{Using Technology to Teach Technology}

Communication technology today provides the networking approach that enables technical professionals and managers to share educational resources on a global scale. Starting in the mid-1960s, instructional television (ITV) in the United States has grown steadily so that, today, it has become the primary way engineers and technical managers continue their education while at work. Nowhere has this been more evident than in the National Technological University (NTU).

A 1992 study reported that NTU provided 78 percent of all university instruction delivered by satellite in North America, and a similar customer-driven initiative delivers non-credit specific development programs to more than 100,000 enrollees each year. Clearly, innovative use of the communication media is upon us with a structure and technology of what has been termed "a virtual university" (Baldwin 1997).

Regional ITV systems operated by individual universities continue to grow, and these networks 
since 1985 have been simultaneously interconnected nationally by satellite via NTU.

The National Technological University is a cooperative effort of 46 major engineering and management colleges linked by satellite and compressed digital-video technology to provide 25,000 hours of instruction to 115,000 participants annually. The regional interactive television systems operated by the individual universities are simultaneously interconnected through NTU. All of its programs focus strongly on learner needs, and it is an accredited and respected university.

The opportunities for more learners and the promise of improved quality of education for everyone is exciting. But excitement about the opportunity to use new media can be a distraction from choosing the most suitable alternatives.

\section{Choosing from the Technology Menu}

In the United States today, the engineering profession has become concerned about the impact of technology on university level learning. In 1900, most engineering graduates entered the workforce with only a baccalaureate degree. This has not changed despite the enormous complexity and rapid change of evolving technology. Once they enter the workforce, engineers face accelerating change that makes career-long learning a necessity. In pursuit of this career-long learning, engineers have become exposed and acclimated to ITV as a learning medium.

Missing from today's distance education research is hard data relating to how the Continuing Professional Educators go about choosing which communication media is most appropriate for their uses. Decision theory has a strong foundation in statistics and the behavioral sciences. By identifying those elements that go to make up decision theory, it is possible to describe the elements that are common to all decisions.

It would seem that the decision-making process is a sequential process of steps that begins with a statement of the problem and ends with an assessment of the results. This assumes that the decision-making process is rational and follows pre-described steps.

-. identifying the problem,

-. developing alternative solutions,

-. evaluating alternatives,

-. choosing the best alternative,

-. implementing the decision, and

-. assessing the results.

There is a large body of literature pertaining to the behavioral theory of decision-making that can attempt to answer this question. Comparing and Contrasting three major decision-making models portrays various ways in which decision-making is perceived and interpreted First, the Rational model selects from among thoroughly analyzed alternatives. All choices, individual and organizational are for the benefit of the entire organization, though evaluation criteria which was developed early in the process, with maximum emphasis placed on logical thinking. 
Assumptions explicit in the rational model are: complete information about alternatives is available, the alternatives can be ranked according to objective criteria, and the alternative selected will provide maximum positive gain for the organization. Implicit assumptions are that ethical dilemmas are non-existent and that the means-end principle and utilitarian principle will dominate ethical considerations.

Second, the Bounded Rationality model tends to be less idealistic, and to consider the limitations of day-to-day decision-making. It reflects individual tendencies to conduct limited searches for alternatives, to make choices under situations of inadequate information or control, and to (satisfice) select the less than best solution. Satisficing is the practice of selecting an acceptable goal, which might be easier to identify, less controversial, and otherwise safer than the best possible solution, very often, the manager's view of being reasonable to achieve. The bounded rationality process reflects the human tendencies to 1) satisfice 2) conduct limited searches, and 3) function with inadequate information and control when making decisions.

Finally, the Political model is evident when bias is employed and the distribution of power and self-serving interests dominate. Deception is a common tactic and distorted figures used to carry out the preferences determined at the outset. These preferences seldom change even as the analysis uncovers new information; definitions, searches, and information exchange are merely methods used to carry out the bias. This method does not allow for ethical dilemmas, but draws upon the principles of hedonism, or might-equals-right, to bluff or take advantages of legal practices, cultures or customs.

However useful each of these models is, they have been found lacking in real world situations in that they tend to be prescriptive. That is, they suggest how decision-makers ought to make decisions, rather than reflecting on how the decisions are made.

On the whole, research on decision-making by individuals has relied largely on analyzing thought processes while solving simplified problems. This theory has not been carried out in practice. In addition, research on decision-making by groups has been concerned with the interactions among the participants in an oversimplified laboratory situation, and not with the decision process. The fallacy in this type of group research is that the very structure of the process has been ignored (Mintzberg, 1976).

Decisions, whether by groups or by individuals, have been categorized as being either routine and structured, or novel and unstructured. In business situations, routine decisions are dealt with using policies, rules and procedures. On the other hand, decisions that are unstructured are usually complex, and do not fit a particular pattern. Business situations like venturing into new processes, products, equipment or markets are unique, and are not dealt with in a routine manner. Relatively little is known about this type of human decision process even though they are the complex decisions that require general problem solving, judgment, intuition, and creativity techniques.

In reality, simplistic models are seldom encountered. More likely, the elements of decision making are found in a common base within an obviously complex structure. 


\section{The Structure Of Unstructured Decisions}

A much more useful model for current purposes is the structured model of the unstructured decision by Henry Mintzberg and his students. Empirical evidence was collected over five years by more than 50 teams of management graduate students. Each team studied for three to six months analyzing 25 different strategic decision processes. From this technique, a general model of unstructured decision-making process was developed. It is a flow chart of seven steps inside three identifiable phases; operating within an environment of three supporting routines and six sets of dynamic elements. Using this general model, the author was able to categorize seven types of path configurations which fit all types of unstructured decisions (Mintzberg, 1976).

Mintzberg brought the elements of the unstructured decision-making process to a common base. The model delineates three distinct phases of the strategic decision process: I) Identification, 2) Development, and 3) Selection; and describes the phases in a mainline of seven central routines: 1) Decision Recognition, 2) Diagnosis, 3) Search, 4) Design, 5) Screen, 6) Evaluation-Choice, and 7) Authorization. Important to the model are supporting routines and dynamic elements. These routines are imbedded in an environment of three Supporting Routines, and six sets of Dynamic Factors, all consisting of human elements.

This general model, obviously complex, is the result of rigorous and intense analysis and study of the decision-making process. Its application has withstood scrutiny and review. Selecting an alternative is not simply choosing the one or best solution; it is a complete phase in the process considering screening, evaluation, and choice. Selecting is an integral part of the process within the Evaluation-Choice Routine, and has been defined as "crude, at best" in the literature, and loaded with soft human elements, particularly in bargaining, design, and development.

Mintzberg demonstrated that complex and dynamic decision processes could be conceptually structured. He described elements and routines about which little is known, concluding that selection routines are mere trimmings in the overall decision process. Ultimately, it is the Decision Support Routines that make up the totality of impact on the process. They are off the mainline on the flow chart, wherein soft issues guide all three of the Decision Supporting routines. Thereupon, it is the human elements that make up the totality of the dynamics of the overall scheme, to include the decision control routine, the decision communications routine, and the political routine. Furthermore, the Dynamic Factors are primarily human elements acting upon the total process, and not just upon any single element.

Large sections of the general model are moderated by human elements. My questionnaire will come from the theoretical model. There is a gap in the literature about how these decisions are made. They are not made how some statistician say they are made. I intend to use develop a questionnaire to test the model.

Likewise, behavioral influences such as individual values, personalities, propensity for risk, potential for disagreement, and ethical intensity all come to bear. The question is, "How do Continuing Professional Engineering Educators go about dealing with the human elements when selecting technology to teach technology?" 
They focus largely on technical issues. They rarely or only marginally consider the non-technical or human elements, which ultimately influence levels of acceptance and effectiveness of the chosen technologies on the part of teachers and participants.

What understanding do they have, or sense do they make of these elements regarding the organizational issues of competition, politics, power and influence? Exploring the beliefs, actions and reflections of decision-makers will provide insight into how the individual and the organization are integrated.

VII. The Inquiry

Data to answer this question will be collected by a survey of key decision-makers in a purposive sample of 23 leading high tech Fortune 500 companies. The questionnaire will ask open-ended questions directed at business-based managers and leaders such as: vice-president of human resources, director of human resource development, director of organizational development, manager of professional and management development, personnel manager, etc. The written survey will ask to consider all human, economic, and environmental elements in addition to engineering and professional considerations. Follow up will by telephone and personal interviews for in-depth exploration, and for ranking in order of importance.

This structured qualitative study of key decision makers in industry will be conducted through survey techniques of focus group interviews, biographical data/reflection questionnaires, and personal telephone interviews to provide in-depth detail-rich data based on individual perspectives and experiences. The decision-makers I intend to study are Continuing Professional Engineering Educators and officers at Fortune-500 companies represented on the National Technological University (NTU) Advisory Committee. All are involved in decisionmaking regarding the planning and programming of corporate engineering development programs.

The first step is to invite them to participate. Following this step, a select focus group of 3 or 4 will be used to identify relevant elements for inclusion in a survey questionnaire. At that point, I am going to talk to them about how they went through the selection process in choosing the system they are now using. Possible beginning questions may include: "Is the media selected on the basis of defined learning needs? Instructional needs? Professional or technical expertise? Available budget? Is the delivery system selected because it is more glamorous or more expensive? What are the criteria for selection?"

The survey questionnaire will involve open-ended questions about communication technologies. The questions will define which technologies are perceived to be the best match for delivering externally provided competency education, as well as the concerns, reservations, and challenges currently known and foreseen. Reference will be made to a leadership model that focuses on the role played by leaders in making selection decisions.

Telephone interviews will follow the survey to elicit responses ranked in order of importance. Interviewees will be asked to react to responses provided by the survey and to indicate the extent 
of agreement with the mean agreement scores of all other respondents. Finally, results will be compiled, analyzed, and summarized into a discussion of the findings' implications, which will then be distributed for comment to all participating organizations.

\section{Conclusion}

Themes that emerge from the study may include: forces driving change, shared vision, opportunities for change, effect of outsiders on the decision team, nature of resistance, moving from or toward central control, redesigning processes, personal stresses, costs, relationships and connections, hidden agendas, role of human resource professionals, or others.

As higher education goes beyond its traditional boundaries to create useful and meaningful programs with Continuing Professional Engineering Educators, both must be prepared to create an awareness of these external influences. It is expected that many of these elements do not align with what adult educators emphasize in instructional design and delivery, suggesting a more holistic perspective on issues of program planning.

Defining the decision-making profile of corporations and developing a strategy to address the profile elements will go a long way to helping you seek the understanding you can take to your professions and to those administrators and decision makers through your educational programs.

Bibliography

1. Queeney, Donna S. (1996). Continuing Professional Education. The ASTD Training \& Development Handbook, Fourth Edition, R.L. Craig, ed. New York: McGraw-Hill.

2. Knowles, Malcolm; The Modern Practice of Adult Education, Cambridge Books, New York, 1980.

3. Baldwin, Lionel V.; (1997, July). The National Technological University: A Pioneering "Virtual University", Lecture presented at Tokyo Institute of Technology, Tokyo, Japan.

4. Mintzberg, H., Raisinghani, D., \& Theoret, A. (1976). The Structure of "Unstructured" Decisions. Administrative Science Quarterly, 21, 266-275.

PETER J. GRAYBASH, JR.

Pete Graybash is an industrial engineer with 39 years of experience in engineering, manufacturing, and continuing professional education. Most recently he served as engineering education manager at AMP Inc., advocating worldwide engineering development. Previous experience includes engineering management positions with P\&S Syracuse Electronics, Schick Electric and Armstrong. He is a doctoral candidate in Adult Education at Penn State. and Armstrong. He is a doctoral candidate in Adult Education at Penn State. 